\title{
Exosomes in Head and Neck Squamous Cell Carcinoma
}

\author{
Cheng Xiao ${ }^{1}$, Fang Song ${ }^{2}$, Yu Long Zheng ${ }^{1}$, Jiong $L^{3}{ }^{3}$, Qiang Feng Wang ${ }^{1}$ and Nong $X u^{1 *}$ \\ ${ }^{1}$ Department of Medical Oncology, College of Medicine, The First Affiliated Hospital, Zhejiang University, Hangzhou, China, \\ ${ }^{2}$ Department of Anesthesiology, College of Medicine, The First Affiliated Hospital, Zhejiang University, Hangzhou, China, \\ ${ }^{3}$ Department of Oral and Maxillofacial Surgery, College of Medicine, The First Affiliated Hospital, Zhejiang University, \\ Hangzhou, China
}

OPEN ACCESS

Edited by:

Cheng-Chia Yu,

Chung Shan Medical

University, Taiwan

Reviewed by:

Augusto Schneider, Universidade Federal de

Pelotas, Brazil

Yin-Hwa Eva Shih

Asia University, Taiwan

${ }^{*}$ Correspondence:

Nong Xu

nongxu@zju.edu.cn

Specialty section:

This article was submitted to

Head and Neck Cancer,

a section of the journal

Frontiers in Oncology

Received: 25 May 2019

Accepted: 27 August 2019

Published: 18 September 2019

Citation:

Xiao C, Song F, Zheng YL, LV J, Wang QF and Xu N (2019) Exosomes

in Head and Neck Squamous Cell

Carcinoma. Front. Oncol. 9:894.

doi: 10.3389/fonc.2019.00894
Exosomes are small membranous vesicles that contain proteins, lipids, genetic material, and metabolites with abundant information from parental cells. Exosomes carry and deliver bioactive contents that can reprogram the functions of recipient cells and modulate the tumor microenvironment to induce pathological events through cell-to-cell communication and signal transduction. Tumor-derived exosomes (TDEs) in head and neck squamous cell carcinoma (HNSCC) are involved in most aspects of cancer initiation, invasion, progression, immunoregulation, therapeutic applications, and treatment resistance. In addition, HNSCC-derived exosomes can be used to obtain information on diagnostic and therapeutic biomarkers in circulating blood and saliva. Currently, the biology, mechanisms, and applications of TDEs in HNSCC are still unclear, and further research is required. In this review, we discuss various aspects of exosome biology, including exosomal components, exosomal biomarkers, and molecular mechanisms involved in immunoregulation, cancer metastasis, and therapy resistance. We also describe recent applications to update our understanding of exosomes in HNSCC.

Keywords: exosomes, head and neck squamous cell carcinoma, tumor microenvironment, biomarkers, therapy resistance

\section{INTRODUCTION}

Head and neck cancer (HNC) is one of the most widespread malignancies worldwide. Although continual progress has been made in the treatment of HNC, the 5-year overall survival rate of advanced HNC remains low at approximately 50\% $(1,2)$. HNC frequently develops from mucosal surfaces of the mouth, including the oral cavity (tongue, lip, buccal, gingiva, and palate), oropharynx, larynx, and perioral skin carcinoma (3). More than $90 \%$ of HNCs are head and neck squamous cell carcinoma (HNSCC). The exact etiology of HNSCC remains unclear; however, tobacco and alcohol consumption are major risk factors for HNSCC, as demonstrated in epidemiological studies. Mucosal human papilloma virus (HPV) is also related to a subset of HNSCCs; $25.9 \%$ of HNSCCs are HPV positive, whereas the prevalence of HPV in oropharyngeal squamous cell carcinoma (SCC) is $34.1 \%$, which is higher than that in oral SCC (4).

Exosomes, which were first discovered in 1983, are 30-150 nm mature double membrane multivesicular bodies (MVBs) originating from the endosomal pathway (5). Exosomes exist in the extracellular space and in liquids, such as blood, urine, and saliva (6). Exosomes are associated with many physiologic aspects of the disease via intercellular communication and signal transduction, indicating that exosomes have potential clinical applications as biomarkers 
and therapeutic targets. Tumor-derived exosomes (TDEs) contain a cytomembrane, proteins, nucleic acids, lipids, and other substances from parental tumor cells (7). Moreover, exosomes are known to be involved in nearly all stages of cancer (8-11). Growing evidence has demonstrated that TDEs participate in the development, progression, and treatment of cancer by mediating intercellular communication and signal transduction $(12,13)$. The bioactive components of HNSCCderived exosomes, such as microRNAs, transcription factors, and oncogenic proteins, play key roles in mediating tumorigenesis, tumor microenvironment reprogramming, immune tolerance, promoting metastasis, and therapy resistance. For example, intracellular annexin 1 (ANXA1) regulates epidermal growth factor receptor (EGFR) activity and alter the release of EGFR-containing TDEs in HNCs (14). Exosomes produced by hypoxic oral SCC cells deliver viral $m i R-21$ to normoxic cells, inducing the epithelial-mesenchymal transition (EMT) to promote cell migration and invasion (15). Another study showed that exosomal nuclear factor- $\kappa \mathrm{B}$-activating kinaseassociated protein 1 (NAP1) derived from oral cancer promotes the cytotoxicity of natural killer (NK) cells via activation of the interferon regulatory factor (IRF-3) signaling pathway in recipient cells (16). In addition, $m i R-34 a-5 p$ in cancerassociated fibroblast (CAF)-derived exosomes in oral SCC stimulates the proliferation and metastasis of oral cancer cells through the AKT/glycogen synthease kinase- $3 \beta / \beta$-catenin/Snail signaling cascade (17). A recent study demonstrated that thrombospondin 1 derived from oral SCC exosomes is also involved in the polarization of macrophages to M1-like tumorassociated macrophages and promotes the invasion of cancer cells (18). HNSCC-derived exosomes containing EphrinB1 may manipulate the tumor microenvironment through induction of tumor innervation (19). Additionally, Sento demonstrated that oral SCC-derived exosomes promote tumor growth by activating the phosphatidylinositol 3-kiase (PI3K)/AKT, mitogen-activated protein kinase (MAPK)/extracellular signal-regulated kinase (ERK), and c-Jun N-terminal kinase-1/signal transducer and activator of transcription (STAT) 2 pathways (20). Emerging evidence has supported the vital role of TDEs in the development, progression, and treatment of HNSCC.

In this review, we summarize many aspects of exosome biology and functions in HNSCC.

\section{BIOGENESIS, FEATURES, AND COMPONENTS OF EXOSOMES}

\section{Inward Budding and MVB Formation}

Different types of vesicles, including extracellular vesicles (EVs), MVBs, and exosomes, have been described and often labeled interchangeably in many previous studies. Although these different types of vesicles share overlapping features, they have distinct morphologies, properties, biogenesis mechanisms, and functional roles. Plasma membrane components and enclosing cytosolic components are incorporated into the invaginating membrane, resulting in the formation of early endosomes (21). Exosomes typically originate from inward budding from the membrane and are then released into the extracellular space via activation of $\mathrm{Ca}^{2+}$-dependent or Rab-GTPases (22). Briefly, exosomes are generated from early endosomes, mature into MVBs, and are then secreted into the extracellular space upon fusion with the plasma membrane. First, exosomes start as early endosomes, which are formed by endocytosis of the plasma membrane. The biogenesis of exosomes and sorting of functional cargo is precisely regulated by certain mechanisms involving multiple factors. The most commonly described pathway for exosomes biogenesis is the endosomal sorting complex required for transport (ESCRT) machinery. Four types of ESCRTs (ESCRT-0-III) are involved in regulating MVB formation, vesicle budding, and protein cargo sorting (23). The ESCRT mechanism is initiated and sequestrated by ubiquitinated proteins to domains of the endosomal membrane via ubiquitin binding subunits of ESCRT-0 in the endosomal membrane, then interacting with the ESCRT-I and ESCRTII complexes inducing membrane deformation into buds. Finally, the ESCRT-III complex separates from the MVBs membrane (23-25). However, the machinery that drives the load of protein cargo into ESCRT-dependent exosomes is still unclear.

Cells also utilize ESCRT-independent pathways, involving insphingosine-1-phosphate, ceramide, tetraspanin-enriched microdomains, and sphingomyelinase, for exosome production and release (26-28). These ESCRT-independent mechanisms may participate in promoting domain-induced budding, sorting of bioactive molecules into exosomes, segregation of cargo within the endosomal membrane, and exosome formation.

The ESCRT-dependent and -independent mechanisms of exosome release are based on the cell origin. In addition, membrane proteins of lysosomes and late endosomes may be important for the biogenesis and secretion of exosomes (29).

\section{Regulated Secretion and Intercellular Interactions}

Exosome secretion is involved in various signaling pathways. For example, the key regulatory role of $\mathrm{RAB}$ family proteins in trafficking intracellular exosomes was demonstrated by Colombo et al. (30). Another report showed that the Wnt pathway is particularly important for the dysregulation of exosome release in cancer cells (31). Additionally, the secretion of exosomes is mediated through exocytosis-associated molecular motors and cytoskeletal proteins (32). Spontaneous secretion of exosomes usually occurs at the steady state; however, some conditions are known to stimulate exosomes. Indeed, cell intrinsic signals are known to enhance the release of high levels of TDEs from cancer cells via activation of oncogenic signaling pathways or regulation of membrane fusion machinery (33). In addition, evidence suggests that microenvironmental conditions enhance exosome release from cancer cells $(15,34)$. Exosomes are then released into the extracellular environment through exocytosis or degraded by fusing with lysosomes. As previously described, Rab GTPases are essential regulators of exosome secretion. Furthermore, several studies have shown that soluble $\mathrm{N}$-ethylmaleimide-sensitive component attachment protein receptor complexes, which are 
involved in membrane fusion machinery, may affect the secretion of exosomes (35-37).

Ultimately, exosomes are internalized by recipient cells through receptor-mediated endocytosis, pinocytosis, phagocytosis, or fusion with the cell membrane, resulting in delivery of molecular and genetic components into the recipient cells (30). Exosomes target recipient cells after secretion into the extracellular space and then induce changes in downstream signaling pathways. The specificity of target recipient cells is dependent on the type of ligand/receptor pairs present on exosomes and recipient cells; and a study provides further insight that glycans are key players in the process of exosomes uptake (38). A schematic representation of exosome biogenesis and secretion pathways is shown in Figure 1.

\section{FEATURES AND COMPONENTS OF EXOSOMES}

Typical exosomes exhibit a particular biconcave or cup-like shape and are observed as spheroids under transmission electron microscopy (39). Exosome contents are rich in nucleic acids, cytosolic/endosome proteins, and cytoskeleton components, which have unique biological activities (40). The components of TDEs are distinct from those of exosomes released from healthy cells. TDEs deliver functional cargo, including oncogenes and oncogenic proteins that can exert biological activities. The functional cargoes, such as protein cargo, RNA cargo, and DNA cargo, of TDEs in the tumor microenvironment have an important role in promoting cancer progression and metastasis (Figure 1) (33). Protein cargoes, including different oncoproteins, immunomodulatory molecules, and growth factors, act as mediators of tumorigenesis. TDEs also contain abundant nucleic acids, such as microRNAs (miRNAs), mRNA, long noncoding RNAs, and DNAs, which deliver genetic information (41-43).

Many researchers have analyzed the functions of miRNAs in exosomes, and various specific miRNA cargoes and extracellular components have been detected in exosomes. These observations highlight the specificity of exosomal miRNAs, distinct miRNA signatures of exosomes, and various functions of exosomal miRNAs. A series of experiments have analyzed the function of the miRNAs in exosomes since their initial description (44). It has been reported that there was a selection of specific miRNA cargoes and extracellular exports in exosomes (30). These observations highlight the specificity of exosomal miRNAs, distinct miRNA signatures of exosomes, and various functions of exosomal miRNAs. Exosomal miRNAs are associated with a variety of pathological activities, including tumorigenesis, invasion, progression, angiogenesis, metastasis,

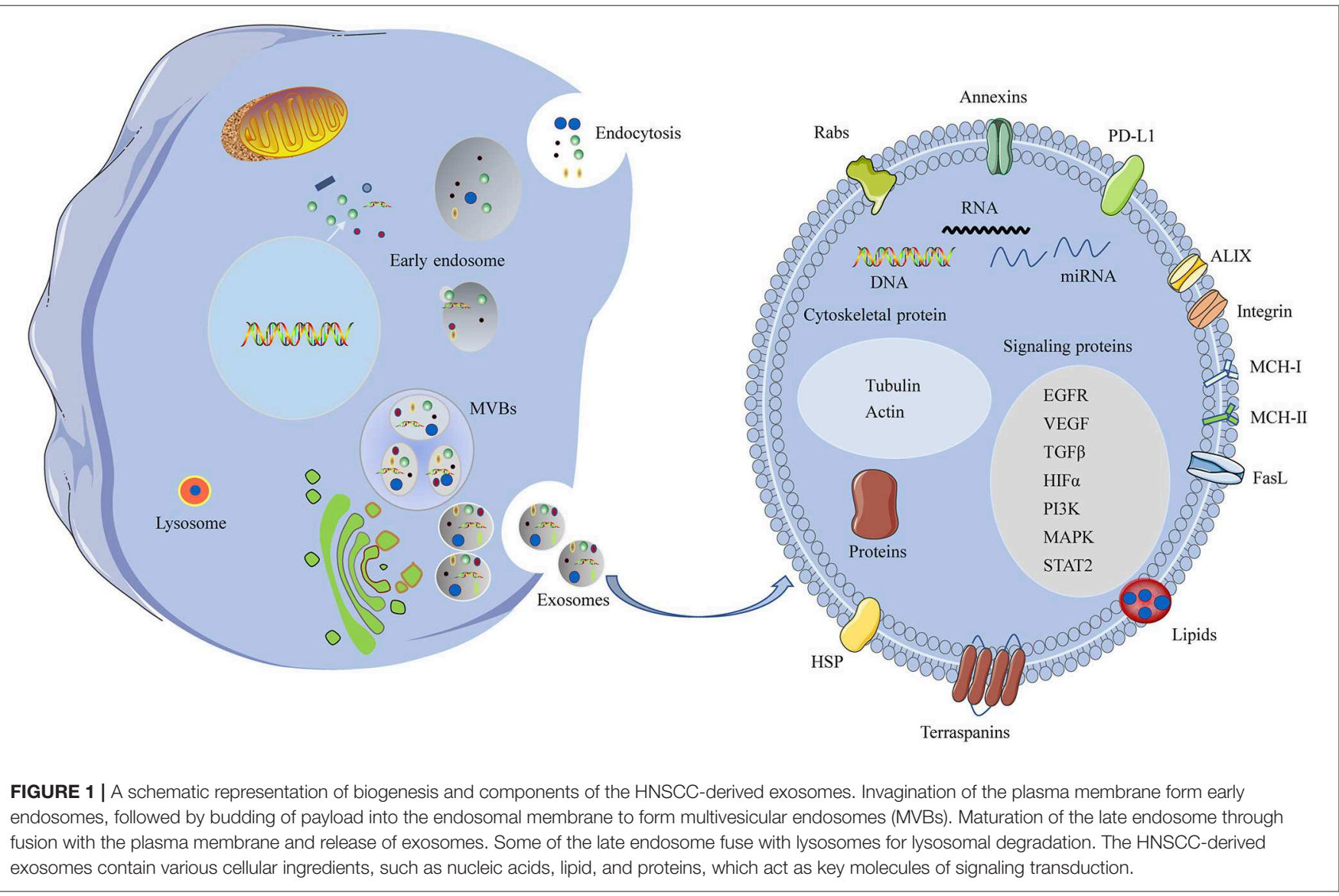


and chemoresistance. However, not all exosomal miRNAs are implicated in tumor-supportive mechanisms. miRNAs of exosomes are highly functional, with roles in intercellular communication and tumor microenvironment regulation, indicating that exosomal miRNAs play important roles in diagnostic and therapeutic applications.

In addition, exosomes contain ceramides, lipids, sphingolipids, cholesterol, and glycerophospholipids. Because exosomes originate from the fusion of endocytic compartments with the plasma membrane, the protein, lipid, and double lipid layer compositions can be used to identify exosomes. The most commonly identified markers are ALIX and tetraspanins, such as CD9, CD63, CD81, and CD82 (23).

\section{FUNCTIONS OF EXOSOMES IN HNSCC}

Exosomes have been identified as bioactive and informative nano-sized MVBs that influence many aspects of the development and progression of HNSCC. Here, we discuss recent findings of the mechanisms through which HNSCCderived exosomes modulate the immune response, tumor microenvironment, cell-to-cell communication, and tumor invasion. We also describe exosomes as potential biomarkers and discuss their applications in cancer therapy and therapy resistance.

\section{EXOSOMES AS POTENTIAL BIOMARKERS}

In the clinical setting, some relevant biomarkers have been shown to influence treatments for patients with HNSCC. Several reports have shown that liquid biopsies, including biopsies of circulating tumor DNA, circulating tumor cells, and exosomal miRNAs, can have potential clinical applications in $\mathrm{HNC}$ (45). Moreover, HPV status is a prognostic factor in HNSCC; patients with HPV positivity have better responsiveness to chemotherapy and radiotherapy and are more susceptible to immune surveillance. In addition, miRNAs are independent prognostic markers for patients with HPV-negative HNSCC (46), and EGFR overexpression is associated with poorer prognosis and outcomes in HNSCC (47). However, further studies are needed to establish biomarkers for staging HNSCC and facilitating therapeutic decision-making.

Exosomes modulate various pathological activities to promote cancer cell growth, invasion, and distant metastasis. TDEs also carry valuable genomic and proteomic information and can provide information regarding alterations in genomic and proteomic profiles of exosomes from patients with cancer in response to anticancer therapies (48). Such proteomic and genetic components are well-protected within the lipid bilayer and can be preserved without significant loss of functional profiling. Therefore, exosomes may serve as promising markers for monitoring cancer progression and therapeutic responses (49).

TDEs can be obtained from blood or saliva of patients with HNSCC, indicating that TDEs may represent a real-time, noninvasive, clinically relevant biomarker of cancer progression, and treatment responses $(45,50-53)$. For example, the number of exosomes in the plasma has been shown to be a prognosis indicator for HNSCC. Gimzewski reported that elevated exosome numbers, exosome sizes, and interexosomes were detected in the saliva of patients with oral cancer (54). In addition, patients with HNSCC with advanced-stage disease and shorter overall survival usually exhibit elevated levels of exosomes in the plasma, indicating that plasma exosomes in HNSCC may have applications in monitoring tumor progression (52, 54). One previous study demonstrated that TDE signatures could serve as candidate biomarkers for early cancer diagnosis, monitoring, and surveillance in HPV-16-associated oropharyngeal (55). Moreover, a non-invasive method involving Fourier-transform infrared spectroscopy of salivary exosomes was shown to have high sensitivity and specificity in the diagnosis of oral cancer (56).

Non-coding RNAs from plasma exosomes have been extensively studied as potential biomarkers in HNSCC because they are derived from whole tumor cells and may therefore represent whole cellular RNAs $(57,58)$. Several studies have reported that cancer cells can selectively pack selected miRNAs into exosomes, and these selective exosomal miRNAs then act as tumor suppressors or oncogenes in HNSCC (59-61). Additionally, a hypoxic microenvironment can stimulate oral SCC to generate $m i R$-21-rich exosomes, which are associated with lymph node metastasis (15). Serum exosomal miR-21 and homeobox transcript antisense RNA (HOTAIR) are also significantly associated with the clinical characteristics of laryngeal SCC (59). In a previous study, elevated CAF-derived exosomal miR-196a levels were shown to be correlated with cisplatin resistance in HNSCC through targeting cyclindependent kinase (CDK) N1B and inhibitor of growth family member 5 (ING5), indicating that this miRNA may serve as a promising predictor of cisplatin resistance and poor survival in HNSCC (60). A study by Zhou and colleagues revealed that there were significant differences in expression between exosomal miRNAs and cellular miRNAs in laryngeal SCC (61). Furthermore, oral cancer-derived salivary exosomal $m i R-512-3 p$ and $m i R-412-3 p$ may serve as potential biomarkers (62). In another example, Inazawa and colleagues found that exosomal miR-1246 induces cell motility and invasion through directly targeting differentially expressed in normal vs. neoplastic/MAPK-activating death domain-containing $2 \mathrm{D}$ in oral SCC (63). Collectively, these results suggested that exosomal miRNAs could serve as excellent diagnostic and prognostic biomarkers.

Analysis of exosomal proteins is a novel tool for developing exosomes as potential biomarkers for HNSCC. More than $80 \%$ of HNSCCs exhibit overexpression of EGFR in the membrane, and hyperactivity EGFR plays an important role in tumorigenesis development and drug-resistance mechanisms by activating various signaling pathways, including the PI3K/AKT, RAS/MEK/ERK, and Janus kinase (JAK)/STAT pathways, in HNSCC. A recent study showed that EGFR can be secreted from cells via the transport of exosomes and that these EGFRcontaining exosomes have the ability to regulate autocrine VEGF production in endothelial cells (64). Exosomal EGFR mediates metastasis and tumor immunity in lung cancer (65). ANXA1, 
a tumor suppressor in HNSCC, regulates EGFR activity and exosomal phospho-EGFR release, revealing that exosomal EGFR and phospho-EGFR may be prognostic biomarkers in HNSCC (14). Additionally, the levels of exosomal EGFR and phosphoEGFR are reduced after cetuximab treatment, indicating that exosomes can serve as biomarkers to monitor cetuximab treatment (66).

Analysis of exosome protein profiles showed that the characteristics and functions of exosomes from HPV-positive/negative HNC differed significantly. HPV-positive exosomes had low p53 levels and did not contain cyclin D1, but did harbor p16, E6/E7, and the T-cell inhibitory protein PTPN11 (67). A recent study revealed that proteome analysis of salivary extracellular vesicles may yield prognostic biomarkers for oral SCC (68).

The microenvironment of HNSCC is highly immunosuppressive, and the programmed cell death (PD)1/PD-ligand 1 (PD-L1) pathway plays an important role in HNSCC. High levels of PD-L1 are associated with poor outcomes in various types of cancer, including HNSCC. PD-1 checkpoint inhibitors were found to be safe and effective in platinum-refractory recurrent or metastatic $\operatorname{HNSCC}(69,70)$. Furthermore, a study by Whiteside and colleagues indicated that $\mathrm{PD}-\mathrm{L}_{1}{ }^{+}$exosomes in the plasma were related to immune suppression and disease progression. Additionally, blocking $\mathrm{PD}-\mathrm{L} 1^{+}$exosome signaling to PD- $1^{+} \mathrm{T}$ cells attenuated immune suppression in patients with HNSCC (71). In another study, Ferris reported that JAK2/STAT1 signaling was involved in EGFR-mediated immune evasion in HNSCC and that therapies targeting this signaling pathway may be beneficial for blocking PD-L1 upregulation in HNSCC (72). Moreover, elevated heatshock protein 90 levels in TDEs have been reported to serve as potential biomarkers for clinical stage and prognosis in patients with oral cancer (73). A recent study showed that plasmaderived exosomes were associated with disease progression of HNSCC after separation into $\mathrm{CD}^{+}$and $\mathrm{CD}^{-}$fractions (74). Furthermore, HNSCC-derived exosomes have been shown to exhibit synergistic interactions with invadopodia, indicating that exosomes play key roles in promoting cancer invasion (35). TDEs inducing transcriptome reprogramming can cause cancerassociated pathologies in HNSCC, including angiogenesis, immunoregulation, and metastasis; these functional differences in HNSCC may serve as candidate markers (75).

A growing body of evidence has shown that the characteristics of TDEs and exosomal components (e.g., exosomal miRNAs, exosomal proteins) may serve as potential noninvasive biomarkers for the detection, monitoring, and treatment of HNSCC (Figure 2). However, methods for TDE isolation and separation are complicated and time-consuming, and additional studies of TDEs as non-invasive biomarkers are needed.

\section{ROLES IN REGULATING THE TUMOR MICROENVIRONMENT}

\section{Immune Cells}

The tumor microenvironment is formed by stromal cells and is associated with malignant progression (76). Moreover, TDEs play important roles in regulating the tumor microenvironment of HNC (77). The immunological activities of exosomes are related to many aspects of immune regulation, including antigen presentation, immune activation, immune surveillance, and immune suppression. Tumor-infiltrating myeloid-derived suppressor cells (MDSCs), tumor-associated macrophages (TAMs), and regulatory $\mathrm{T}$ cells (Tregs) are known mediators of the immunosuppressive microenvironment and limit the efficacy of immune therapy in HNC (78). Exosomes that contain immunosuppressive molecules can facilitate immunosuppression in cancer, which helps cancer cells escape from immune responses, thereby promoting tumorigenesis (79, 80). Additionally, exosome-associated bioactive proteins and RNAs have been shown to regulate the immune system $(81,82)$. Exosomes may mediate immune suppression through

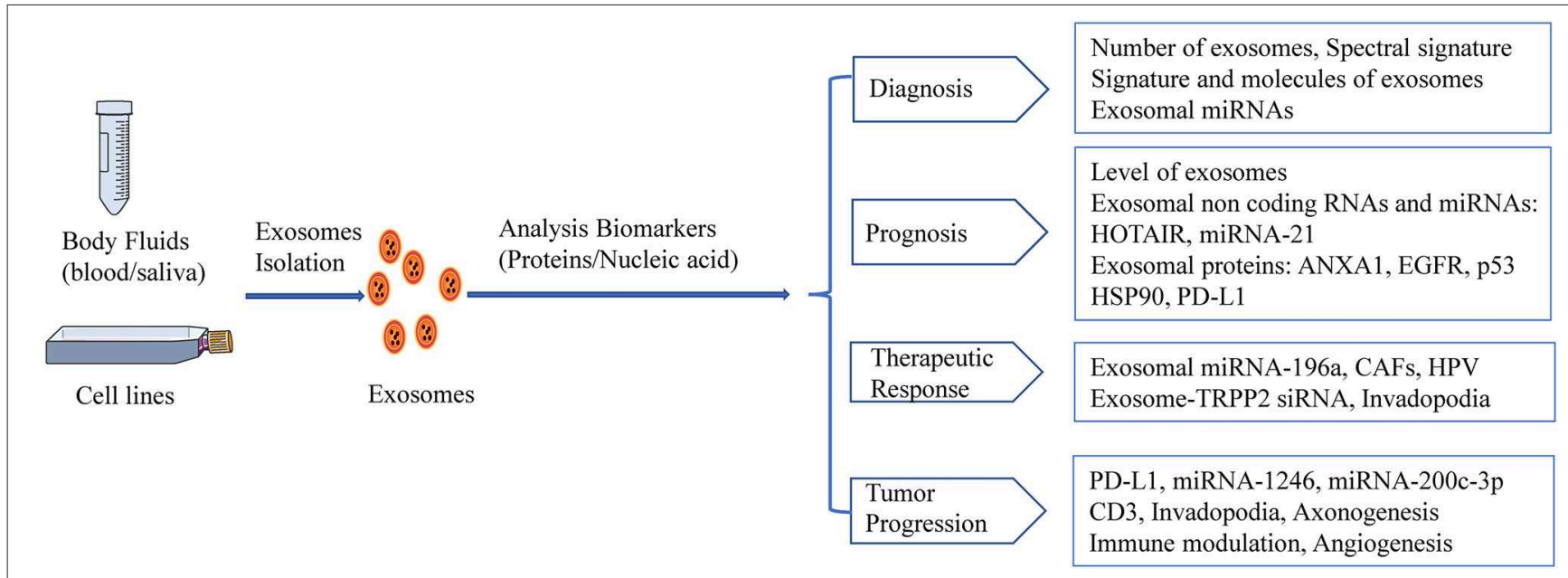

FIGURE 2 | HNSCC-derived exosomes act as biomarkers. Exosomal biomarkers screening of diagnostics, prognosis, or therapeutics in HNSCC. 
directly/indirectly inhibiting the functions of $\mathrm{T}$ cells and NK cells and then altering the number or activity of immune suppressor cells, including MDSCs, Tregs, and HLA-DR cells (83). Exosomes are also involved in different soluble factors such as check-point receptor ligands (PD-L1), inhibitory cytokines (IL-10 and TGF- $\beta 1$ ), death receptor ligands (FasL), ectoenzymes, and prostaglandin E2 responsible for antitumor immunity in the tumor microenvironment $(84,85)$. In lymphocytes and NK cells, exosomes are associated with disease stage and activity in patients with $\mathrm{HNC}$, suggesting that plasma exosomes may be related to HNC progression (86). In cancer, exosome signaling may affect the immune system by inhibiting the maturity of antigen-presenting cells and TDEs that carry and transfer tumor antigens to antigen-presenting cells, thereby inducing $\mathrm{T}$ cell- or NK cell-dependent immune responses (87). In a similar study, TDEs containing FasL and tumor necrosis factor $\alpha$ were found to induce T-cell apoptosis, thereby establishing an immunosuppressive tumor microenvironment to support tumor progression (88). TDEs derived from multiple HNCC cell lines induce a suppressive phenotype in $\mathrm{CD} 8^{+} \mathrm{T}$ cells through galectin-1, indicating that tumor-derived immunosuppressive exosomes may be potential therapeutic targets for preventing T-cell dysfunction and enhancing antitumor immune responses (89). As previously mentioned, the levels of oral SCC-derived $\mathrm{FasL}^{+}$microvesicles are correlated with tumor burden, and the $\mathrm{FasL}^{+}$microvesicles are involved in mediating apoptosis in activated $\mathrm{T}$ lymphocytes via receptor and mitochondrial pathways (51). A recent study showed that $\mathrm{CD} 4{ }^{+} \mathrm{CD} 39^{+}$Tregs produce adenosine by exposure to $\mathrm{CD} 39^{+} \mathrm{CD} 73^{+}$exosomes from plasma in patients with HNSCC, thereby supporting tumor immune escape (90). Additionally, a recent study showed that oxygen pressure regulates the tumor microenvironment by altering exosomal miRNAs, which subsequently regulate the $m i R-21 /$ phosphatase and tensin homolog (PTEN)/PD-L1 axis (91).

\section{HPV-Positive and -Negative Exosomes}

The molecular and functional profiles of exosomes from HPVpositive and -negative HNSCC are different. An early study demonstrated that HNSCC-derived exosomes have different effects on the immune system in HPV-positive and -negative HNSCC. However, there are no differences in suppressive $\mathrm{CD}^{+}{ }^{+}$and $\mathrm{CD}^{+}{ }^{+} \mathrm{T}$ cells between HPV-positive and -negative exosomes, although the responses of human monocyte-derived dendritic cells (DCs) and mature DCs to exosomes are different. HPV-positive exosomes promote DC maturation, whereas HPV-negative exosomes suppress DC maturation. HPV-negative exosomes suppress the expression of antigen processing machinery, whereas HPV-positive exosomes do not (67). HPV-positive exosomes that promote DC maturation may also stimulate antitumor immune responses, thereby improving clinical outcomes in patients with HPV-positive HNSCC (67). HPV has been shown to utilize host exosomes for cell-cell communication and to induce EGFR expression and AKT signaling in recipient cells. One study found that exosomal NAP1 derived from oral cancer cells can promote the activation of
NK cells by increasing the expression and phosphorylation of IRF-3 (92).

\section{Fibroblasts}

CAFs are particularly important for regulating tumor progression. Mesenchymal stem cells reprogrammed by TDEs mediate pro-angiogenic activity and convert stromal cells into CAFs; these cells are a major component of the tumor microenvironment and play key roles in promoting tumor progression (93). HNC-derived CAFs are innately resistant to cisplatin. Indeed, a study by Zhang showed that exosomal miR-196a derived from HNC causes cisplatin resistance by targeting CDKN1B and ING5, highlighting the roles of CAFderived exosomal $m i R-196 a$ in promoting cell proliferation and inhibiting cell apoptosis in the HNC microenvironment (60). Moreover, high expression of microfibril associated protein 5 in CAF-derived exosomes may contribute to the proliferation and metastasis of oral SCC via activation of the MAPK and AKT signaling pathways (94). Additionally, TDEs have been shown to deliver caveolin-1 to the tumor microenvironment to mediate the EMT and CAFs in tongue SCC (95). In a similar study, CAF-derived exosomal $m i R-34 a-5 p$ was found to be associated with oral cancer cell proliferation and metastasis in oral SCC (17). Thus, TDEs are emerging as potent mediators of the tumor microenvironment in HNSCC (Figure 3).

\section{ROLES OF TDES IN REGULATING CANCER PROGRESSION AND METASTASIS}

Many studies have evaluated the roles of TDEs in cancer initiation and progression. Numerous molecules in TDEs have been implicated in the initiation and progression of cancer cells or the tumor microenvironment (96). Indeed, TDEs can transform epithelial cells into cancerous cells, thereby initiating tumorigenesis. Studies have shown that TDEs reprogram the functions of recipient cells and facilitate premetastatic niche transformation to promote metastasis through cell-cell communication or autocrine signals. Additionally, TDEs facilitate tumor progression by delivery of factors necessary for sustaining tumor growth via utilizing autocrine or juxtacrine signaling (97). Furthermore, many studies have shown that RNA from TDEs can promote metastasis (57). The shuttling of miRNA molecules may cause tumorigenesis, tumor progression, and mRNA entry into recipient cells, resulting in protein translation and metastasis (44). The EMT has been implicated in cancer cell progression and metastasis, and TDEs deliver functional complexes by membrane fusion with recipient cells and binding of the recipient cell membrane receptors to promote the EMT (98).

Exosomes derived from hypoxic oral SCC cells promote cell migration and invasion by delivering $m i R-21$ to normoxic cells in HNSCC (15). Nakashima conducted a study indicating that $m i R-200 c-3 p$ had invasive capacity in the human oral SCC microenvironment (99). Moreover, CAFs contribute to the proliferation and metastasis of oral cancer cells via 


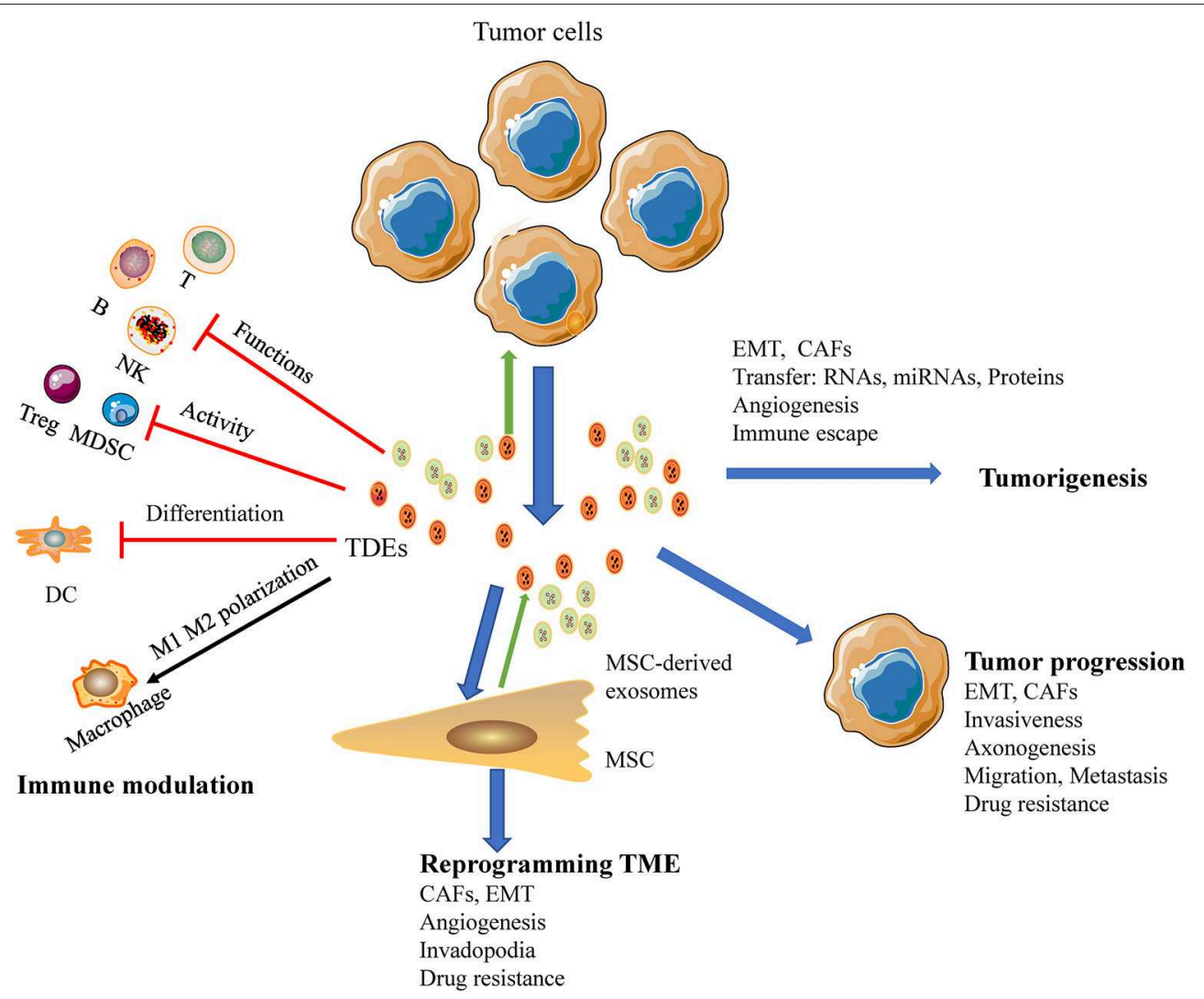

FIGURE 3 | A macroscopic view of the functions of HNSCC-derived exosomes in the tumor microenvironment. Exosomes reduce tumor immunosurveillance by interfering with the immune system: (a) inhibit the functions of immune cells; (b) suppress the activity of Tregs, MDSCs; (c) interference the differentiation of DC; (d) TAMs polarization. HNSCC-derived exosomes mediated metastasis via stimulating proliferation of endothelial cells, EMT, CAFs, and promote angiogenesis as well as re-programming of the stromal compartment. Also HNSCC-derived exosomes influence cancer progression, promoting cancer cell growth, and invasive behavior of cancer cells and development of therapy resistance.

exosome-mediated paracrine miR-34a-5p signaling (17). Several studies have reported the heterogeneity of transforming growth factor (TGF) $\beta$ signaling in oral cavity SCC. TGF $\beta$ expressed on the surface of TDEs can differentiate fibroblasts into myofibroblasts, thereby promoting tumor progression and metastasis in oral cavity SCC (100). Additionally, exosomes target different organs via variations in integrin molecules expressed on the surface. Released exosomes can be delivered to distant organs to promote oncogenic activity by constructing a suitable premetastatic tumor microenvironment for tumor migration. Thus, TDEs contribute to the development of this premetastatic niche and induce metastatic potential in recipient cells. A recent study showed that exosomes can transport EGFR to the liver to remodel the liver microenvironment (101). TDEs can transfer oncogenic EGFR to endothelial cells, triggering upregulation of vascular endothelial growth factor (VEGF) and autocrine VEGF in endothelial cells exposed to cancer cell-related MVs (64). Moreover, oral cancer cellderived MVs promote endothelial cell angiogenesis through the Shh/RhoA signaling pathway (102). Additional experiments have demonstrated that exosomal miR-150 promotes tumorigenesis by upregulating VEGF, and another study showed that HNSCC-derived exosomes stimulate angiogenesis in vitro and in vivo through functional reprogramming of endothelial cells (103). These studies suggest that exosomal miRNA could affect the biology of endothelial cells; then the exosomal miRNA induces angiogenesis in HNC through different regulation pathways.

TDEs modulate the immune response in tumor microenvironment interactions implicated in cancer progression. Oral SCC-derived exosomes induced M1-like TAMs polarized and promoted tumor metastasis (18). And tumor released exosomes containing EphrinB1 potentiate induce axonogenesis will promote tumor innervation in HNSCC (19). And the delivery of miRNA-21-abundant exosomes promote EMTmediated M2-like polarization of TAMs may promote tumor progression of HNSCC (104). Another study demonstrated that exosomes derived from irradiated HNSCC cells can modify cancer cell movement and promote migration of recipient cells through AKT-signaling (105). In an additional series of experiments, exosomes were recruited to the plasma membrane of invadopodia, and knockdown of Rab27a decreased exosome secretion and extracellular matrix digestion associated with maturing invadopodia (35). The mechanisms through which TDEs regulate cancer progression and metastasis are illustrated in Figure 3. 


\section{THERAPEUTIC APPLICATION}

Exosomes are cell-derived nanoparticles that have unique properties, such as low immunogenicity, strong ability to cross physiological barriers, good biodistribution and bioavailability, and reduced immunogenicity. Thus, TDEs may have important roles as potential vehicles for anticancer drugs. In addition, exosomes can be remodeled through their parental cells or supplemented with desired biological activity (106). Exosomes have been designed as promising therapeutic agents in the treatment of various cancers (107-109). Because patients with metastatic HNSCC have a poor prognosis and do not typically respond well to traditional therapies, efficient targeted delivery of conventional chemotherapeutic drugs may be facilitated by innovative approaches to engineering drug delivery systems, such as exosomes.

Drug carrier exosomes have been isolated from various types of cells, including HEK-293 cells, immature DCs, macrophages, and cancer cells. Different therapeutic agents, including proteins, small interfering RNAs (siRNAs), miRNAs, and targeted drugs, can be incorporated into exosomes via electroporation, chemicalbased transfection, modification of parental cells, or direct incubation, thereby increasing bioactivity and achieving targeted delivery in patients. The James Graham Brown Cancer Center initiated a phase I clinical trial to test the therapeutic effects of plant exosomes in HNC (NCT01668849). In various types of cancer, exosomes have been shown to shuttle miRNAs and soluble proteins as therapeutic molecules into recipient cells and tissues $(107,110)$. A hypoxic microenvironment promotes the generation of $m i R$-21-rich exosomes by oral SCC in a hypoxia-inducible factor (HIF)-1a- and HIF-2a-dependent manner, and these $m i R$-21-rich exosomes then mediate migration and invasion behaviors. Restoration of miR-21 expression in HIF1a- and HIF-2a-depleted exosomes rescues oral SCC migration and invasion (15). Moreover, hypoxic TDEs mediate MDSC function through the $m i R-21 / \mathrm{PTEN} / \mathrm{PD}-\mathrm{L} 1$ axis in oral SCC (91). These findings indicate the therapeutic value of exosome inhibition for oral SCC treatment.

Anti-EGFR nanobodies anchored on extracellular vesicles via glycosyl phosphatidylinositol may improve the targeting ability of extracellular vesicles, highlighting the potential applications of these extracellular vesicles as a drug delivery system and new tool in EGFR-expressing tumor cells (111). The bloodbrain barrier restricts drugs from entering into the brain, which can reduce the therapeutic effects of brain cancer treatments. Brain endothelial cell-derived exosomes can deliver anticancer drugs across the blood-brain barrier for the treatment of brain cancer. Additionally, exosome-based drug carriers can mediate permeability across the blood-brain barrier, enabling the drug to target cancer cells (112).

The main rational approaches for inhibiting exosomemediated tumor-promoting potential have focused on blocking exosome release and suppressing the communication of tumor cells with recipient cells. TDEs are known to be abundantly secreted from cancer cells, making them potential targets for anticancer therapy. Direct targeting of the exosome release process has also been studied for the treatment of cancer.
Exosome-delivered transient receptor potential polycystic 2 (TRPP2) siRNA markedly suppresses TRPP2 expression and inhibits the EMT, suggesting that exosome-TRPP2 siRNA may be an effective RNA-based gene therapy in the treatment of HNC (113). Invadopodia also enhance exosome secretion; accordingly, silencing the expression of invadopodia may suppress TDE biogenesis and release (114). Another potential strategy for exosomal dysregulation is the inhibition of exosome uptake. Treatment with heparin blocks the uptake of exosomes by oral SCC cells, thereby attenuating exosome-induced cancer progression to inhibit the growth and progression of oral SCC cells (20). However, the use of these reagents for eliminating exosomes is currently limited to research use only because these reagents may also induce off-target effects. Further studies are needed to explore the translational implications of exosometargeted reagents.

Owing to the potential presence of TDEs and the unique biomarkers associated with these vesicles, TDEs may also have applications as vaccine immunotherapies (115). Antigenpresenting exosomes from B lymphocytes and DCs containing MHCI/II complexes could stimulate $\mathrm{CD} 4^{+}$and $\mathrm{CD} 8^{+} \mathrm{T}$ cells as therapeutic HPV vaccines (116). Additionally, HPV oncogenes play vital roles in HPV-induced carcinogenesis, and silencing of endogenous HPV E6/E7 expression affects both the contents and levels of MVs released from HPV-positive cancer cells (117). These findings indicate that inhibition of endogenous HPV E6/E7 expression may have therapeutic applications. HPV vaccines based on endogenously engineered exosomes for HNC have been evaluated in several phase I clinical trials (118). However, additional studies are required to determine the feasibility and safety of TDEs as cancer vaccines. One of the major challenges in developing this approach is establishing scalable, reproducible methods for exosome production. MSCs may have uses in exosome production at a clinically applicable scale owing to their ability of produce large amounts of exosomes.

Collectively, these studies suggest that exosome-based strategies may have many benefits over conventional drug regimens; however, there are some limitations and challenges to the use of exosomes. First, because TDEs contain genetic components from cancer cells, cancer cells may not be suitable parental cells for exosome targeting in clinical applications. Additionally, efficient loading of exosomes without significant alterations to the structure and content of exosomal membranes may be difficult. Overall, these reports indicate that exosomes may function as exceptional gene delivery vectors that are safe, efficient, organ-/cell-specific, and nonimmunogenic. Nevertheless, significant efforts are required to before clinical applications are feasible.

\section{ANTI-CANCER THERAPY RESISTANCE}

Current therapy options for HNSCC include surgery, radiotherapy, chemotherapy, anti-EGFR-antibody treatment, and immunotherapy (119). However, drug resistance remains a major obstacle for achieving successful curative treatment of cancer. Drug resistance includes endogenous drug resistance, 
innate drug resistance, and acquired drug resistance. Acquired drug resistance is a process through which cancer cells exposed to chemotherapy, radiation, or targeted therapy show reprogramming of their genome to acquire resistance to the therapy. Cancer cells may mitigate the effects of radiation and chemotherapy through different mechanisms $(120,121)$.

Given the important roles of exosomes in cellular communication and the tumor microenvironment, many studies have indicated that exosomes are involved in anticancer therapy resistance. In one mechanism, exosomes sequester cytotoxic drugs in intracellular vesicles and subsequently negate the effects of drugs within the cells (122). Owing to the nature of exosomes as mediators of cell-cell communication in the tumor microenvironment, these exosomes play important roles in therapy resistance by transferring various contents, such as miRNAs, mRNAs, DNAs, and proteins, to induce extrinsic therapy resistance (123). Similarly, exosomes also promote therapy resistance by transferring mRNAs, miRNAs, and other components. In addition, TDEs mediate therapy resistance by different mechanism, including improved DNA repair, anti-apoptotic signaling, or delivery of transporters to treatment-sensitive cells. As mediators of mesenchymal stem cells and the EMT, exosomes also promote tumor microenvironment-associated treatment resistance (123).

Exosomes derived from drug-resistant cancer cells mediate drug resistance through direct shuttling of drugs out of the cells (124). The AKT pathway is a frequently mutated oncogenic pathway in HNSCC and functions as a key regulator of radiation resistance and a major driver of cellular movement and migration (125). Exosomes derived from irradiated HNSCC cells trigger the AKT pathway to promote migration and increase chemotaxis in recipient cancer cells (105). Radiation therapy may increase the invasive and metastatic properties of HNSCC via release of an abundance of exosomes in hypoxic cancer tissue after radiotherapy (126-128). In patients with melanoma receiving PD-1 blockade therapy, the level of circulating exosomal PD-L1 correlates with tumor burden and response to therapy (129). The PD-1/PD-L1 immune checkpoint signaling axis exhibits remarkable responses in platinumrefractory recurrent or metastatic HNSCC; PD-L1-containing TDEs, which transfer functional PD-L1 and inhibit immune

\section{REFERENCES}

1. Bray F, Ferlay J, Soerjomataram I, Siegel RL, Torre LA, Jemal A. Global cancer statistics 2018: GLOBOCAN estimates of incidence and mortality worldwide for 36 cancers in 185 countries. Cancer J Clin. (2018) 68:394-424. doi: $10.3322 /$ caac. 21492

2. Ang KK, Harris J, Wheeler R, Weber R, Rosenthal DI, Nguyen-Tan PF, et al. Human papillomavirus and survival of patients with oropharyngeal cancer. N Engl J Med. (2010) 363:24-35. doi: 10.1056/NEJMoa091 2217

3. Lydiatt WM, Patel SG, O'Sullivan B, Brandwein MS, Ridge JA, Migliacci JC, et al. Head and neck cancers-major changes in the American Joint Committee on cancer eighth edition cancer staging manual. Cancer J Clin. (2017) 67:122-37. doi: 10.3322/caac. 21389 responses, may be regulators and biomarkers of resistance to PD-1 blockade therapy. In addition, exosomes derived from cisplatin-resistant HNSCC cells deliver $m i R-21$ to parental cells and induce cisplatin resistance, suggesting that these exosomes may function primarily through gene regulation (130). TDEs have been implicated in contributing to drug resistance in HNSCC (Figure 3).

\section{FUTURE IMPLICATIONS}

TDEs contain numerous bioactive cellular molecules and genetic characteristics, enabling them to alter the functions of recipient cells and the tumor microenvironment. Accordingly, TDEs play important regulatory roles in cancer. Indeed, TDEs are involved in many aspects of intercellular substance transmission and signal transfer, contributing to the initiation, development, metastasis, treatment resistance, and immunosuppression of HNSCC. In HNSCC, TDEs may serve as potential clinical biomarkers of progression or responses to therapy owing to their various functional contents (proteins, genes) and elements of the parental cancer cells. Nevertheless, exosomes have not yet been applied in the treatment of HNSCC. Further studies are needed to elucidate the molecular mechanisms involved in the release of exosomes and to explore the clinical applications of these vesicles. Understanding how cancer cells utilize TDEs to promote cancer growth and progression may lead to the development of novel therapies for HNSCC. Therefore, much work is needed to establish exosome-based therapies for the treatment of HNSCC.

\section{AUTHOR CONTRIBUTIONS}

CX, FS, and NX: review concept, review design, interpretation, manuscript preparation, and manuscript review. YZ, JL, and QW: manuscript preparation and manuscript review.

\section{FUNDING}

This study was funded by National Health and Family Planning Commission Research Fund \& Zhejiang Provincial Medical Science and Technology Plan Project (No: KWJ-ZJ-1802).

4. Kreimer AR, Clifford GM, Boyle P, Franceschi S. Human papillomavirus types in head and neck squamous cell carcinomas worldwide: a systematic review. Cancer Epidemiol Biomark Prev. (2005) 14:467-75. doi: 10.1158/1055-9965.EPI-04-0551

5. Pan BT, Johnstone RM. Fate of the transferrin receptor during maturation of sheep reticulocytes in vitro: selective externalization of the receptor. Cell. (1983) 33:967-78. doi: 10.1016/0092-8674(83)90040-5

6. Keller S, Ridinger J, Rupp AK, Janssen JW, Altevogt P. Body fluid derived exosomes as a novel template for clinical diagnostics. J Transl Med. (2011) 9:86. doi: 10.1186/1479-5876-9-86

7. Xin T, Greco V, Myung P. Hardwiring stem cell communication through tissue structure. Cell. (2016) 164:1212-25. doi: 10.1016/j.cell.2016.02.041

8. Steinbichler TB, Dudas J, Riechelmann H, Skvortsova, II. The role of exosomes in cancer metastasis. Semin Cancer Biol. (2017) 44:170-81. doi: 10.1016/j.semcancer.2017.02.006 
9. Becker A, Thakur BK, Weiss JM, Kim HS, Peinado H, Lyden D. Extracellular vesicles in cancer: cell-to-cell mediators of metastasis. Cancer Cell. (2016) 30:836-48. doi: 10.1016/j.ccell.2016.10.009

10. Liu Y, Cao X. Organotropic metastasis: role of tumor exosomes. Cell Res. (2016) 26:149-50. doi: 10.1038/cr.2015.153

11. Zhang Y, Wang XF. A niche role for cancer exosomes in metastasis. Nat Cell Biol. (2015) 17:709-11. doi: 10.1038/ncb3181

12. Zhang HG, Grizzle WE. Exosomes: a novel pathway of local and distant intercellular communication that facilitates the growth and metastasis of neoplastic lesions. Am J Pathol. (2014) 184:28-41. doi: 10.1016/j.ajpath.2013.09.027

13. Wang Z, Chen JQ, Liu JL, Tian L. Exosomes in tumor microenvironment: novel transporters and biomarkers. J Transl Med. (2016) 14:297. doi: 10.1186/s12967-016-1056-9

14. Raulf N, Lucarelli P, Thavaraj S, Brown S, Vicencio JM, Sauter T, et al. Annexin A1 regulates EGFR activity and alters EGFR-containing tumourderived exosomes in head and neck cancers. Eur J Cancer. (2018) 102:52-68. doi: 10.1016/j.ejca.2018.07.123

15. Li L, Li C, Wang S, Wang Z, Jiang J, Wang W, et al. Exosomes derived from hypoxic oral squamous cell carcinoma cells deliver miR-21 to normoxic cells to elicit a prometastatic phenotype. Cancer Res. (2016) 76:1770-80. doi: 10.1158/0008-5472.CAN-15-1625

16. Wang $\mathrm{Y}$, Qin $\mathrm{X}$, Zhu $\mathrm{X}$, Chen $\mathrm{W}$, Zhang J, Chen $\mathrm{W}$. Oral cancer-derived exosomal NAP1 enhances cytotoxicity of natural killer cells via the IRF-3 pathway. Oral Oncol. (2018) 76:34-41. doi: 10.1016/j.oraloncology.2017.11.024

17. Li YY, Tao YW, Gao S, Li P, Zheng JM, Zhang SE, et al. Cancer-associated fibroblasts contribute to oral cancer cells proliferation and metastasis via exosome-mediated paracrine miR-34a-5p. EBioMedicine. (2018) 36:209-20. doi: 10.1016/j.ebiom.2018.09.006

18. Xiao M, Zhang J, Chen W, Chen W. M1-like tumor-associated macrophages activated by exosome-transferred THBS1 promote malignant migration in oral squamous cell carcinoma. J Exp Clin Cancer Res. (2018) 37:143. doi: 10.1186/s13046-018-0815-2

19. Madeo M, Colbert PL, Vermeer DW, Lucido CT, Cain JT, Vichaya EG, et al. Cancer exosomes induce tumor innervation. Nat Commun. (2018) 9:4284. doi: 10.1038/s41467-018-06640-0

20. Sento S, Sasabe E, Yamamoto T. Application of a persistent heparin treatment inhibits the malignant potential of oral squamous carcinoma cells induced by tumor cell-derived exosomes. PLoS ONE. (2016) 11:e0148454. doi: 10.1371 /journal.pone. 0148454

21. Abels ER, Breakefield XO. Introduction to extracellular vesicles: biogenesis, RNA cargo selection, content, release, and uptake. Cell Mol Neurobiol. (2016) 36:301-12. doi: 10.1007/s10571-016-0366-z

22. Blott EJ, Griffiths GM. Secretory lysosomes. Nat Rev Mol Cell Biol. (2002) 3:122-31. doi: 10.1038/nrm732

23. Colombo M, Moita C, van Niel G, Kowal J, Vigneron J, Benaroch P, et al. Analysis of ESCRT functions in exosome biogenesis, composition and secretion highlights the heterogeneity of extracellular vesicles. J Cell Sci. (2013) 126 (Pt 24):5553-65. doi: 10.1242/jcs. 128868

24. Henne WM, Buchkovich NJ, Emr SD. The ESCRT pathway. Dev Cell. (2011) 21:77-91. doi: 10.1016/j.devcel.2011.05.015

25. Katzmann DJ, Babst M, Emr SD. Ubiquitin-dependent sorting into the multivesicular body pathway requires the function of a conserved endosomal protein sorting complex, ESCRT-I. Cell. (2001) 106:145-55. doi: 10.1016/S0092-8674(01)00434-2

26. Airola MV, Hannun YA. Sphingolipid metabolism and neutral sphingomyelinases. Handb Exp Pharmacol. (2013) 215:57-76. doi: 10.1007/978-3-7091-1368-4_3

27. Castro BM, Prieto M, Silva LC. Ceramide: a simple sphingolipid with unique biophysical properties. Prog Lipid Res. (2014) 54:53-67. doi: 10.1016/j.plipres.2014.01.004

28. Perez-Hernandez D, Gutierrez-Vazquez C, Jorge I, Lopez-Martin S, Ursa A, Sanchez-Madrid F, et al. The intracellular interactome of tetraspaninenriched microdomains reveals their function as sorting machineries toward exosomes. J Biol Chem. (2013) 288:11649-61. doi: 10.1074/jbc.M112.445304
29. Zhu H, Guariglia S, Yu RY, Li W, Brancho D, Peinado H, et al. Mutation of SIMPLE in charcot-marie-tooth 1c alters production of exosomes. Mol Biol Cell. (2013) 24:1619-37, S1-3. doi: 10.1091/mbc.e12-07-0544

30. Colombo M, Raposo G, Thery C. Biogenesis, secretion, and intercellular interactions of exosomes and other extracellular vesicles. Annu Rev Cell Dev Biol. (2014) 30:255-89. doi: 10.1146/annurev-cellbio-101512-122326

31. Ekstrom EJ, Bergenfelz C, von Bulow V, Serifler F, Carlemalm E, Jonsson G, et al. WNT5A induces release of exosomes containing pro-angiogenic and immunosuppressive factors from malignant melanoma cells. Mol Cancer. (2014) 13:88. doi: 10.1186/1476-4598-13-88

32. Vlassov AV, Magdaleno S, Setterquist R, Conrad R. Exosomes: current knowledge of their composition, biological functions, and diagnostic and therapeutic potentials. Biochim Biophys Acta. (2012) 1820:940-8. doi: 10.1016/j.bbagen.2012.03.017

33. Bebelman MP, Smit MJ, Pegtel DM, Baglio SR. Biogenesis and function of extracellular vesicles in cancer. Pharmacol Ther. (2018) 188:1-11. doi: 10.1016/j.pharmthera.2018.02.013

34. Wang T, Gilkes DM, Takano N, Xiang L, Luo W, Bishop CJ, et al. Hypoxiainducible factors and RAB22A mediate formation of microvesicles that stimulate breast cancer invasion and metastasis. Proc Natl Acad Sci USA. (2014) 111:E3234-42. doi: 10.1073/pnas.1410041111

35. Hoshino D, Kirkbride KC, Costello K, Clark ES, Sinha S, Grega-Larson N, et al. Exosome secretion is enhanced by invadopodia and drives invasive behavior. Cell Rep. (2013) 5:1159-68. doi: 10.1016/j.celrep.2013.10.050

36. Verweij FJ, Bebelman MP, Jimenez CR, Garcia-Vallejo JJ, Janssen H, Neefjes J, et al. Quantifying exosome secretion from single cells reveals a modulatory role for GPCR signaling. J Cell Biol. (2018) 217:1129-42. doi: $10.1083 /$ jcb. 201703206

37. Wei $\mathrm{Y}$, Wang $\mathrm{D}$, Jin F, Bian $\mathrm{Z}$, Li L, Liang $\mathrm{H}$, et al. Pyruvate kinase type M2 promotes tumour cell exosome release via phosphorylating synaptosome-associated protein 23. Nat Commun. (2017) 8:14041. doi: $10.1038 /$ ncomms 14041

38. Williams C, Pazos R, Royo F, Gonzalez E, Roura-Ferrer M, Martinez A, et al. Assessing the role of surface glycans of extracellular vesicles on cellular uptake. Sci Rep. (2019) 9:11920. doi: 10.1038/s41598-019-48499-1

39. Yellon DM, Davidson SM. Exosomes: nanoparticles involved in cardioprotection? Circ Res. (2014) 114:325-32. doi: 10.1161/CIRCRESAHA.113.300636

40. Thery C, Boussac M, Veron P, Ricciardi-Castagnoli P, Raposo G, Garin J, et al. Proteomic analysis of dendritic cell-derived exosomes: a secreted subcellular compartment distinct from apoptotic vesicles. J Immunol. (2001) 166:7309-18. doi: 10.4049/jimmunol.166.12.7309

41. Zhou W, Fong MY, Min Y, Somlo G, Liu L, Palomares MR, et al. Cancer-secreted miR-105 destroys vascular endothelial barriers to promote metastasis. Cancer Cell. (2014) 25:501-15. doi: 10.1016/j.ccr.2014.03.007

42. Melo SA, Sugimoto H, O'Connell JT, Kato N, Villanueva A, Vidal A, et al. Cancer exosomes perform cell-independent microRNA biogenesis and promote tumorigenesis. Cancer Cell. (2014) 26:707-21. doi: 10.1016/j.ccell.2014.09.005

43. Wilusz JE, Sunwoo H, Spector DL. Long noncoding RNAs: functional surprises from the RNA world. Genes Dev. (2009) 23:1494-504. doi: 10.1101/gad.1800909

44. Valadi H, Ekstrom K, Bossios A, Sjostrand M, Lee JJ, Lotvall JO. Exosome-mediated transfer of mRNAs and microRNAs is a novel mechanism of genetic exchange between cells. Nat Cell Biol. (2007) 9:654-9. doi: $10.1038 /$ ncb1596

45. Nonaka T, Wong DTW. Liquid biopsy in head and neck cancer: promises and challenges. J Dental Res. (2018) 97:701-8. doi: $10.1177 / 0022034518762071$

46. Hess J, Unger K, Maihoefer C, Schuttrumpf L, Wintergerst L, Heider T, et al. A five-microRNA signature predicts survival and disease control of patients with head and neck cancer negative for HPV infection. Clin Cancer Res. (2019) 25:1505-16. doi: 10.1158/1078-0432.CCR-18-0776

47. $\mathrm{Xu} \mathrm{MJ}$, Johnson DE, Grandis JR. EGFR-targeted therapies in the post-genomic era. Cancer Metastasis Rev. (2017) 36:463-73. doi: $10.1007 / \mathrm{s} 10555-017-9687-8$ 
48. Whiteside TL. The potential of tumor-derived exosomes for noninvasive cancer monitoring. Expert Rev Mol Diagnos. (2015) 15:1293-310. doi: 10.1586/14737159.2015.1071666

49. Wortzel I, Dror S, Kenific CM, Lyden D. Exosome-mediated metastasis: communication from a distance. Dev Cell. (2019) 49:347-60. doi: 10.1016/j.devcel.2019.04.011

50. Principe S, Hui AB, Bruce J, Sinha A, Liu FF, Kislinger T. Tumor-derived exosomes and microvesicles in head and neck cancer: implications for tumor biology and biomarker discovery. Proteomics. (2013) 13:1608-23. doi: $10.1002 /$ pmic. 201200533

51. Kim JW, Wieckowski E, Taylor DD, Reichert TE, Watkins S, Whiteside TL. Fas ligand-positive membranous vesicles isolated from sera of patients with oral cancer induce apoptosis of activated T lymphocytes. Clin Cancer Res. (2005) 11:1010-20.

52. Theodoraki MN, Hoffmann TK, Jackson EK, Whiteside TL. Exosomes in HNSCC plasma as surrogate markers of tumour progression and immune competence. Clin Exp Immunol. (2018) 194:67-78. doi: 10.1111/cei.13157

53. Zlotogorski-Hurvitz A, Dayan D, Chaushu G, Salo T, Vered M. Morphological and molecular features of oral fluid-derived exosomes: oral cancer patients versus healthy individuals. J Cancer Res Clin Oncol. (2016) 142:101-10. doi: 10.1007/s00432-015-2005-3

54. Sharma S, Gillespie BM, Palanisamy V, Gimzewski JK. Quantitative nanostructural and single-molecule force spectroscopy biomolecular analysis of human-saliva-derived exosomes. Langmuir. (2011) 27:14394-400. doi: $10.1021 / \mathrm{la} 2038763$

55. Kannan A, Hertweck KL, Philley JV, Wells RB, Dasgupta S. Genetic mutation and exosome signature of human papilloma virus associated oropharyngeal cancer. Sci Rep. (2017) 7:46102. doi: 10.1038/srep46102

56. Zlotogorski-Hurvitz A, Dekel BZ, Malonek D, Yahalom R, Vered M. FTIRbased spectrum of salivary exosomes coupled with computational-aided discriminating analysis in the diagnosis of oral cancer. J Cancer Res Clin Oncol. (2019) 145:685-94. doi: 10.1007/s00432-018-02827-6

57. Momen-Heravi F, Bala S. Emerging role of non-coding RNA in oral cancer. Cell Signal. (2018) 42:134-43. doi: 10.1016/j.cellsig.2017.10.009

58. Rabinowits G, Bowden M, Flores LM, Verselis S, Vergara V, Jo VY, et al. Comparative analysis of microRNA expression among benign and malignant tongue tissue and plasma of patients with tongue cancer. Front Oncol. (2017) 7:191. doi: 10.3389/fonc.2017.00191

59. Wang J, Zhou Y, Lu J, Sun Y, Xiao H, Liu M, et al. Combined detection of serum exosomal miR-21 and HOTAIR as diagnostic and prognostic biomarkers for laryngeal squamous cell carcinoma. Med Oncol. (2014) 31:148. doi: 10.1007/s12032-014-0148-8

60. Qin X, Guo H, Wang X, Zhu X, Yan M, Wang X, et al. Exosomal miR196a derived from cancer-associated fibroblasts confers cisplatin resistance in head and neck cancer through targeting CDKN1B and ING5. Genome Biol. (2019) 20:12. doi: 10.1186/s13059-018-1604-0

61. Huang Q, Yang J, Zheng J, Hsueh C, Guo Y, Zhou L. Characterization of selective exosomal microRNA expression profile derived from laryngeal squamous cell carcinoma detected by next generation sequencing. Oncol Rep. (2018) 40:2584-94. doi: 10.3892/or.2018.6672

62. Gai C, Camussi F, Broccoletti R, Gambino A, Cabras M, Molinaro $\mathrm{L}$, et al. Salivary extracellular vesicle-associated miRNAs as potential biomarkers in oral squamous cell carcinoma. BMC Cancer. (2018) 18:439. doi: $10.1186 / \mathrm{s} 12885-018-4364-\mathrm{z}$

63. Sakha S, Muramatsu T, Ueda K, Inazawa J. Exosomal microRNA miR1246 induces cell motility and invasion through the regulation of DENND2D in oral squamous cell carcinoma. Sci Rep. (2016) 6:38750. doi: 10.1038/srep38750

64. Al-Nedawi K, Meehan B, Kerbel RS, Allison AC, Rak J. Endothelial expression of autocrine VEGF upon the uptake of tumor-derived microvesicles containing oncogenic EGFR. Proc Natl Acad Sci USA. (2009) 106:3794-9. doi: 10.1073/pnas.0804543106

65. Huang SH, Li Y, Zhang J, Rong J, Ye S. Epidermal growth factor receptorcontaining exosomes induce tumor-specific regulatory $\mathrm{T}$ cells. Cancer Invest. (2013) 31:330-5. doi: 10.3109/07357907.2013.789905

66. van Dommelen SM, van der Meel R, van Solinge WW, Coimbra M, Vader P, Schiffelers RM. Cetuximab treatment alters the content of extracellular vesicles released from tumor cells. Nanomedicine. (2016) 11:881-90. doi: 10.2217/nnm-2015-0009

67. Ludwig S, Sharma P, Theodoraki MN, Pietrowska M, Yerneni SS, Lang $S$, et al. Molecular and functional profiles of exosomes from $\mathrm{HPV}(+)$ and HPV(-) head and neck cancer cell lines. Front Oncol. (2018) 8:445. doi: $10.3389 /$ fonc. 2018.00445

68. Winck FV, Prado Ribeiro AC, Ramos Domingues R, Ling LY, Riano-Pachon $\mathrm{DM}$, Rivera C, et al. Insights into immune responses in oral cancer through proteomic analysis of saliva and salivary extracellular vesicles. Sci Rep. (2015) 5:16305. doi: 10.1038/srep16305

69. Ferris RL, Blumenschein G Jr, Fayette J, Guigay J, Colevas AD, Licitra L, et al. Nivolumab for recurrent squamous-cell carcinoma of the head and neck. $N$ Engl J Med. (2016) 375:1856-67. doi: 10.1056/NEJMoa1602252

70. Chow LQM, Haddad R, Gupta S, Mahipal A, Mehra R, Tahara M, et al. Antitumor activity of pembrolizumab in biomarker-unselected patients with recurrent and/or metastatic head and neck squamous cell carcinoma: results from the phase Ib KEYNOTE-012 expansion cohort. J Clin Oncol. (2016) 34:3838-45. doi: 10.1200/JCO.2016.68.1478

71. Theodoraki MN, Yerneni SS, Hoffmann TK, Gooding WE, Whiteside TL. Clinical significance of PD-L1(+) exosomes in plasma of head and neck cancer patients. Clin Cancer Res. (2018) 24:896-905. doi: 10.1158/1078-0432.CCR-17-2664

72. Concha-Benavente F, Srivastava RM, Trivedi S, Lei Y, Chandran U, Seethala RR, et al. Identification of the cell-intrinsic and -extrinsic pathways downstream of EGFR and IFNgamma that induce PD-L1 expression in head and neck cancer. Cancer Res. (2016) 76:1031-43. doi: 10.1158/0008-5472.CAN-15-2001

73. Ono K, Eguchi T, Sogawa C, Calderwood SK, Futagawa J, Kasai T, et al. HSPenriched properties of extracellular vesicles involve survival of metastatic oral cancer cells. J Cell Biochem. (2018) 119:7350-62. doi: 10.1002/jcb.27039

74. Theodoraki MN, Hoffmann TK, Whiteside TL. Separation of plasma-derived exosomes into $\mathrm{CD} 3((+))$ and $\mathrm{CD} 3((-))$ fractions allows for association of immune cell and tumour cell markers with disease activity in HNSCC patients. Clin Exp Immunol. (2018) 192:271-83. doi: 10.1111/cei.13113

75. Qadir F, Aziz MA, Sari CP, Ma H, Dai H, Wang X, et al. Transcriptome reprogramming by cancer exosomes: identification of novel molecular targets in matrix and immune modulation. Mol Cancer. (2018) 17:97. doi: 10.1186/s12943-018-0846-5

76. Quail DF, Joyce JA. Microenvironmental regulation of tumor progression and metastasis. Nat Med. (2013) 19:1423-37. doi: 10.1038/nm.3394

77. Dayan D, Salo T, Salo S, Nyberg P, Nurmenniemi S, Costea DE, et al. Molecular crosstalk between cancer cells and tumor microenvironment components suggests potential targets for new therapeutic approaches in mobile tongue cancer. Cancer Med. (2012) 1:128-40. doi: 10.1002/cam4.24

78. Davis RJ, Van Waes C, Allen CT. Overcoming barriers to effective immunotherapy: MDSCs, TAMs, and Tregs as mediators of the immunosuppressive microenvironment in head and neck cancer. Oral Oncol. (2016) 58:59-70. doi: 10.1016/j.oraloncology.2016.05.002

79. Filipazzi P, Burdek M, Villa A, Rivoltini L, Huber V. Recent advances on the role of tumor exosomes in immunosuppression and disease progression. Semin Cancer Biol. (2012) 22:342-9. doi: 10.1016/j.semcancer.2012.02.005

80. Monypenny J, Milewicz H, Flores-Borja F, Weitsman G, Cheung A, Chowdhury R, et al. ALIX regulates tumor-mediated immunosuppression by controlling EGFR activity and PD-L1 presentation. Cell Rep. (2018) 24:630-41. doi: 10.1016/j.celrep.2018.06.066

81. van der Grein SG, Nolte-'t Hoen EN. "Small Talk" in the Innate immune system via RNA-containing extracellular vesicles. Front Immunol. (2014) 5:542. doi: $10.3389 /$ fimmu.2014.00542

82. Aucher A, Rudnicka D, Davis DM. MicroRNAs transfer from human macrophages to hepato-carcinoma cells and inhibit proliferation. J Immunol. (2013) 191:6250-60. doi: 10.4049/jimmunol.1301728

83. Vincent-Schneider H, Stumptner-Cuvelette P, Lankar D, Pain S, Raposo G, Benaroch P, et al. Exosomes bearing HLA-DR1 molecules need dendritic cells to efficiently stimulate specific T cells. Int Immunol. (2002) 14:713-22. doi: $10.1093 /$ intimm/dxf048

84. Whiteside TL. Exosomes and tumor-mediated immune suppression. J Clin Invest. (2016) 126:1216-23. doi: 10.1172/JCI81136 
85. Shi L, Chen S, Yang L, Li Y. The role of PD-1 and PD-L1 in T-cell immune suppression in patients with hematological malignancies. J Hematol Oncol. (2013) 6:74. doi: 10.1186/1756-8722-6-74

86. Ludwig S, Floros T, Theodoraki MN, Hong CS, Jackson EK, Lang S, et al. Suppression of lymphocyte functions by plasma exosomes correlates with disease activity in patients with head and neck cancer. Clin Cancer Res. (2017) 23:4843-54. doi: 10.1158/1078-0432.CCR-16-2819

87. Wolfers J, Lozier A, Raposo G, Regnault A, Thery C, Masurier C, et al. Tumor-derived exosomes are a source of shared tumor rejection antigens for CTL cross-priming. Nat Med. (2001) 7:297-303. doi: 10.1038/85438

88. Zhang HG, Grizzle WE. Exosomes and cancer: a newly described pathway of immune suppression. Clin Cancer Res. (2011) 17:959-64. doi: 10.1158/1078-0432.CCR-10-1489

89. Maybruck BT, Pfannenstiel LW, Diaz-Montero M, Gastman BR. Tumorderived exosomes induce CD8(+) T cell suppressors. J Immunother Cancer. (2017) 5:65. doi: 10.1186/s40425-017-0269-7

90. Schuler PJ, Saze Z, Hong CS, Muller L, Gillespie DG, Cheng D, et al. Human $\mathrm{CD} 4+\mathrm{CD} 39+$ regulatory $\mathrm{T}$ cells produce adenosine upon co-expression of surface CD73 or contact with CD73+ exosomes or CD73+ cells. Clin Exp Immunol. (2014) 177:531-43. doi: 10.1111/cei.12354

91. Li L, Cao B, Liang X, Lu S, Luo H, Wang Z, et al. Microenvironmental oxygen pressure orchestrates an anti- and pro-tumoral gammadelta $\mathrm{T}$ cell equilibrium via tumor-derived exosomes. Oncogene. (2019) 38:2830-43. doi: 10.1038/s41388-018-0627-z

92. Zhou J, Li M, Lim WQ, Luo Z, Phua SZF, Huo R, et al. A transferrin-conjugated hollow nanoplatform for redox-controlled and targeted chemotherapy of tumor with reduced inflammatory reactions. Theranostics. (2018) 8:518-32. doi: 10.7150/thno.21194

93. Whiteside TL. Exosome and mesenchymal stem cell cross-talk in the tumor microenvironment. Semin Immunol. (2018) 35:69-79. doi: 10.1016/j.smim.2017.12.003

94. Principe S, Mejia-Guerrero S, Ignatchenko V, Sinha A, Ignatchenko A, Shi W, et al. Proteomic analysis of cancer-associated fibroblasts reveals a paracrine role for MFAP5 in human oral tongue squamous cell carcinoma. J Proteome Res. (2018) 17:2045-59. doi: 10.1021/acs.jproteome.7b00925

95. Vered M, Lehtonen M, Hotakainen L, Pirila E, Teppo S, Nyberg P, et al. Caveolin-1 accumulation in the tongue cancer tumor microenvironment is significantly associated with poor prognosis: an in-vivo and in-vitro study. BMC Cancer. (2015) 15:25. doi: 10.1186/s12885-015-1030-6

96. Milane L, Singh A, Mattheolabakis G, Suresh M, Amiji MM. Exosome mediated communication within the tumor microenvironment. J Control Release. (2015) 219:278-94. doi: 10.1016/j.jconrel.2015.06.029

97. Syn N, Wang L, Sethi G, Thiery JP, Goh BC. Exosome-mediated metastasis: from epithelial-mesenchymal transition to escape from immunosurveillance. Trends Pharmacol Sci. (2016) 37:606-17. doi: 10.1016/j.tips.2016.04.006

98. Blackwell RH, Foreman KE, Gupta GN. The role of cancer-derived exosomes in tumorigenicity \& epithelial-to-mesenchymal transition. Cancers. (2017) 9:E105. doi: 10.3390/cancers9080105

99. Kawakubo-Yasukochi T, Morioka M, Hazekawa M, Yasukochi A, Nishinakagawa T, Ono $\mathrm{K}$, et al. miR-200c-3p spreads invasive capacity in human oral squamous cell carcinoma microenvironment. Mol Carcinogenesis. (2018) 57:295-302. doi: 10.1002/mc.22744

100. Languino LR, Singh A, Prisco M, Inman GJ, Luginbuhl A, Curry JM, et al. Exosome-mediated transfer from the tumor microenvironment increases TGFbeta signaling in squamous cell carcinoma. Am J Transl Res. (2016) 8:2432-7.

101. Zhang H, Deng T, Liu R, Bai M, Zhou L, Wang X, et al. Exosome-delivered EGFR regulates liver microenvironment to promote gastric cancer liver metastasis. Nat Commun. (2017) 8:15016. doi: 10.1038/ncomms15016

102. Huaitong X, Yuanyong F, Yueqin T, Peng Z, Wei S, Kai S. Microvesicles releasing by oral cancer cells enhance endothelial cell angiogenesis via Shh/RhoA signaling pathway. Cancer Biol Ther. (2017) 18:783-91. doi: 10.1080/15384047.2017.1373213

103. Ludwig N, Yerneni SS, Razzo BM, Whiteside TL. Exosomes from HNSCC promote angiogenesis through reprogramming of endothelial cells. Mol Cancer Res. (2018) 16:1798-808. doi: 10.1158/1541-7786.MCR-18-0358

104. Hsieh $\mathrm{CH}$, Tai SK, Yang MH. Snail-overexpressing cancer cells promote M2-like polarization of tumor-associated macrophages by delivering MiR-21-abundant exosomes. Neoplasia. (2018) 20:775-88. doi: 10.1016/j.neo.2018.06.004

105. Mutschelknaus L, Azimzadeh O, Heider T, Winkler K, Vetter M, Kell R, et al. Radiation alters the cargo of exosomes released from squamous head and neck cancer cells to promote migration of recipient cells. Sci Rep. (2017) 7:12423. doi: 10.1038/s41598-017-12403-6

106. Batrakova EV, Kim MS. Using exosomes, naturally-equipped nanocarriers, for drug delivery. J Control Release. (2015) 219:396-405. doi: 10.1016/j.jconrel.2015.07.030

107. Yim N, Ryu SW, Choi K, Lee KR, Lee S, Choi H, et al. Exosome engineering for efficient intracellular delivery of soluble proteins using optically reversible protein-protein interaction module. Nat Commun. (2016) 7:12277. doi: 10.1038/ncomms12277

108. Syn NL, Wang L, Chow EK, Lim CT, Goh BC. Exosomes in cancer nanomedicine and immunotherapy: prospects and challenges. Trends Biotechnol. (2017) 35:665-76. doi: 10.1016/j.tibtech.2017. 03.004

109. Wu K, Xing F, Wu SY, Watabe K. Extracellular vesicles as emerging targets in cancer: recent development from bench to bedside. Biochim Biophys Acta Rev Cancer. (2017) 1868:538-63. doi: 10.1016/j.bbcan.2017. 10.001

110. Li L, Piontek K, Ishida M, Fausther M, Dranoff JA, Fu R, et al. Extracellular vesicles carry microRNA-195 to intrahepatic cholangiocarcinoma and improve survival in a rat model. Hepatology. (2017) 65:501-14. doi: 10.1002/hep. 28735

111. Kooijmans SA, Aleza CG, Roffler SR, van Solinge WW, Vader P, Schiffelers RM. Display of GPI-anchored anti-EGFR nanobodies on extracellular vesicles promotes tumour cell targeting. J Extracell Vesicles. (2016) 5:31053. doi: 10.3402/jev.v5.31053

112. Yang T, Martin P, Fogarty B, Brown A, Schurman K, Phipps R, et al. Exosome delivered anticancer drugs across the blood-brain barrier for brain cancer therapy in Danio rerio. Pharm Res. (2015) 32:2003-14. doi: 10.1007/s11095-014-1593-y

113. Wang C, Chen L, Huang Y, Li K, Jinye A, Fan T, et al. Exosome-delivered TRPP2 siRNA inhibits the epithelial-mesenchymal transition of FaDu cells. Oncol Lett. (2019) 17:1953-61. doi: 10.3892/ol.2018.9752

114. Murphy DA, Courtneidge SA. The 'ins' and 'outs' of podosomes and invadopodia: characteristics, formation and function. Nat Rev Mol Cell Biol. (2011) 12:413-26. doi: 10.1038/nrm3141

115. Sharma A, Khatun Z, Shiras A. Tumor exosomes: cellular postmen of cancer diagnosis and personalized therapy. Nanomedicine. (2016) 11:421-37. doi: $10.2217 / \mathrm{nnm} .15 .210$

116. Hao S, Bai O, Li F, Yuan J, Laferte S, Xiang J. Mature dendritic cells pulsed with exosomes stimulate efficient cytotoxic T-lymphocyte responses and antitumour immunity. Immunology. (2007) 120:90-102. doi: 10.1111/j.1365-2567.2006.02483.x

117. Honegger A, Leitz J, Bulkescher J, Hoppe-Seyler K, Hoppe-Seyler F. Silencing of human papillomavirus (HPV) E6/E7 oncogene expression affects both the contents and the amounts of extracellular microvesicles released from HPV-positive cancer cells. Int J Cancer. (2013) 133:1631-42. doi: 10.1002/ijc. 28164

118. Di Bonito P, Accardi L, Galati L, Ferrantelli F, Federico M. Anticancer vaccine for HPV-associated neoplasms: focus on a therapeutic HPV vaccine based on a novel tumor antigen delivery method using endogenously engineered exosomes. Cancers. (2019) 11:E138. doi: $10.3390 /$ cancers 11020138

119. Cramer JD, Burtness B, Le QT, Ferris RL. The changing therapeutic landscape of head and neck cancer. Nat Rev Clin Oncol. (2019). doi: 10.1038/s41571-019-0227-z. [Epub ahead of print]

120. Meads MB, Gatenby RA, Dalton WS. Environment-mediated drug resistance: a major contributor to minimal residual disease. Nat Rev Cancer. (2009) 9:665-74. doi: 10.1038/nrc2714

121. Moitra K, Lou H, Dean M. Multidrug efflux pumps and cancer stem cells: insights into multidrug resistance and therapeutic development. Clin Pharmacol Ther. (2011) 89:491-502. doi: 10.1038/clpt.2011.14

122. Shedden K, Xie XT, Chandaroy P, Chang YT, Rosania GR. Expulsion of small molecules in vesicles shed by cancer cells: association with gene expression and chemosensitivity profiles. Cancer Res. (2003) 63:4331-7. 
123. Steinbichler TB, Dudas J, Skvortsov S, Ganswindt U, Riechelmann H, Skvortsova II. Therapy resistance mediated by exosomes. Mol Cancer. (2019) 18:58. doi: 10.1186/s12943-019-0970-x

124. Namee NM, O’Driscoll L. Extracellular vesicles and anti-cancer drug resistance. Biochim Biophys Acta Rev Cancer. (2018) 1870:123-36. doi: 10.1016/j.bbcan.2018.07.003

125. Bussink J, van der Kogel AJ, Kaanders JH. Activation of the PI3-K/AKT pathway and implications for radioresistance mechanisms in head and neck cancer. Lancet Oncol. (2008) 9:288-96. doi: 10.1016/S1470-2045(08)7 0073-1

126. Pickhard AC, Margraf J, Knopf A, Stark T, Piontek G, Beck C, et al. Inhibition of radiation induced migration of human head and neck squamous cell carcinoma cells by blocking of EGF receptor pathways. BMC Cancer. (2011) 11:388. doi: 10.1186/1471-2407-11-388

127. Beck C, Piontek G, Haug A, Bas M, Knopf A, Stark T, et al. The kallikreinkinin-system in head and neck squamous cell carcinoma (HNSCC) and its role in tumour survival, invasion, migration and response to radiotherapy. Oral Oncol. (2012) 48:1208-19. doi: 10.1016/j.oraloncology.2012. 06.001

128. Mutschelknaus L, Peters C, Winkler K, Yentrapalli R, Heider T, Atkinson $\mathrm{MJ}$, et al. Exosomes derived from squamous head and neck cancer promote cell survival after ionizing radiation. PLOS ONE. (2016) 11:e0152213. doi: 10.1371/journal.pone.0152213

129. Chen G, Huang AC, Zhang W, Zhang G, Wu M, Xu W, et al. Exosomal PD-L1 contributes to immunosuppression and is associated with anti-PD-1 response. Nature. (2018) 560:382-6. doi: 10.1038/s41586-018-0392-8

130. Liu T, Chen G, Sun D, Lei M, Li Y, Zhou C, et al. Exosomes containing miR21 transfer the characteristic of cisplatin resistance by targeting PTEN and PDCD4 in oral squamous cell carcinoma. Acta Biochim Biophys Sin. (2017) 49:808-16. doi: 10.1093/abbs/gmx078

Conflict of Interest Statement: The authors declare that the research was conducted in the absence of any commercial or financial relationships that could be construed as a potential conflict of interest.

Copyright (c) 2019 Xiao, Song, Zheng, Lv, Wang and Xu. This is an open-access article distributed under the terms of the Creative Commons Attribution License (CC $B Y)$. The use, distribution or reproduction in other forums is permitted, provided the original author(s) and the copyright owner(s) are credited and that the original publication in this journal is cited, in accordance with accepted academic practice. No use, distribution or reproduction is permitted which does not comply with these terms. 